\title{
Left Parietal Regions Are Critical for Adaptive Visuomotor Control
}

\author{
Pratik K. Mutha, ${ }^{1,2}$ Robert L. Sainburg, ${ }^{2,3,4}$ and Kathleen Y. Haaland ${ }^{1,5,6}$ \\ ${ }^{1}$ New Mexico Veterans Affairs Healthcare System, Albuquerque, New Mexico 87108, Departments of ${ }^{2}$ Kinesiology and ${ }^{3}$ Neurology and ${ }^{4}$ Program in \\ Neuroscience, Pennsylvania State University, University Park, Pennsylvania 16802, and Departments of ${ }^{5}$ Psychiatry and ${ }^{6}$ Neurology, University of New \\ Mexico, Albuquerque, New Mexico 87131
}

The question addressed in this study is whether parietal brain circuits involved in adaptation to novel visuomotor conditions are lateralized. This information is critical for characterizing the neural mechanisms mediating adaptive behavior in humans, as well as for assessing the effects of unilateral brain damage on function. Moreover, previous research has been controversial in this regard. We compared visuomotor adaptation in 10 patients with focal, unilateral, left or right parietal lesions and healthy control participants. All subjects reached to each of eight targets over three experimental sessions: a baseline session, where the visually displayed and actual hand motion were matched; an adaptation session, where the visual feedback deviated from the actual movement direction by $30^{\circ}$; and an after-effect session, where visual feedback was again matched to hand motion. Adaptation was primarily quantified as a change in initial movement direction throughout the adaptation session and the presence of after-effects when the rotation was removed. Patients with right parietal damage demonstrated normal adaptation and large after-effects, which was comparable to the performance of healthy controls. In contrast, patients with left parietal damage showed a clear deficit in adaptation and showed no after-effects. Thus, our results show that left but not right parietal regions are critical for visuomotor adaptation. These findings are discussed in the context that left parietal regions are critical for the modification of stored representations of the relationship between movement commands and limb and environmental state, as is thought to occur during visuomotor adaptation.

\section{Introduction}

Cerebral lateralization, the functional specialization of the left and right brain hemispheres, has been well characterized for many perceptual and cognitive processes (Gazzaniga, 2000). A large body of work has also investigated the lateralization of movement control mechanisms. These behavioral, neuroimaging, and lesion studies have suggested that each hemisphere might contribute unique mechanisms to the control of movements of either arm (for review, see Sainburg, 2010). However, what these mechanisms are is not yet completely clear. This information is crucial for characterizing the organization of sensorimotor control processes and has significant implications for understanding the effects of unilateral brain damage on function. In this study, we ask whether parietal mecha-

\footnotetext{
Received Dec. 9, 2010; revised March 18, 2011; accepted March 22, 2011.

Author contributions: P.K.M., R.L.S., and K.Y.H. designed research; P.K.M. performed research; P.K.M. analyzed data; P.K.M., R.L.S., and K.Y.H. wrote the paper.

This work was supported by grants to K.Y.H. from the Clinical Science Research and Development Service (101BX007080) and the Rehabilitation Research and Development Service (B4125R) of the Veterans Affairs Office of Research and Development and grants to R.L.S. from the National Institutes of Health (National Institute of Child Health and Human Development) (R01HD39311 and R01HD059783). We thank Jenna Keller, Sierra Widmer, and Melissa Daniels for assistance with data collection; Lee Stapp for help with MRI tracings; Dr. Brad Cushnyr for neuroradiological consultation; Drs. John Adair and Sally Harris and HealthSouth Rehabilitation Hospital and Lovelace Medical Center for patient referral.

Correspondence should be addressed to Dr. Pratik K. Mutha, New Mexico Veterans Affairs Healthcare System, Research Service 151, 1501 San Pedro Drive Southeast, Albuquerque, NM 87108. E-mail: pkm117@psu.edu.

DOI:10.1523/JNEUROSCI.6432-10.2011

Copyright $\odot 2011$ the authors $\quad 0270-6474 / 11 / 316972-10 \$ 15.00 / 0$
}

nisms involved in adaptation to novel visuomotor conditions are lateralized.

Adaptive motor control in humans has been widely studied using prism and virtual reality visuomotor rotation paradigms that introduce a mismatch between the actual and visually displayed limb position. The algorithms used by the nervous system to adapt to these conditions are thought to involve visuomotor "remapping" based on the altered relationship between limb movement and visual feedback. Adaptation thus requires modifications to our prior representations about how movement commands affect the state of the body and the environment (Krakauer, 2009; Shabbott and Sainburg, 2010).

The neural substrates underlying such adaptive control are only beginning to be identified. While adaptation deficits in cerebellar-damaged patients (Martin et al., 1996; Tseng et al., 2007) have demonstrated the importance of the cerebellum in this process, several functional neuroimaging studies have implicated parietal regions as well. However, studies attributing a role for parietal circuits have been controversial, especially with regard to whether parietal regions in the left, right, or both hemispheres are critical for visuomotor adaptation. Whereas early studies emphasized activation of contralateral parietal cortex (Clower et al., 1996), newer studies have reported modulation of only left (Danckert et al., 2008), only right (Ghilardi et al., 2000; Krakauer et al., 2004), or bilateral (Grefkes et al., 2004; Diedrichsen et al., 2005; Graydon et al., 2005) parietal activity during adaptation. Activation of right parietal regions has been proposed to reflect a right hemisphere specialization for visuospatial 

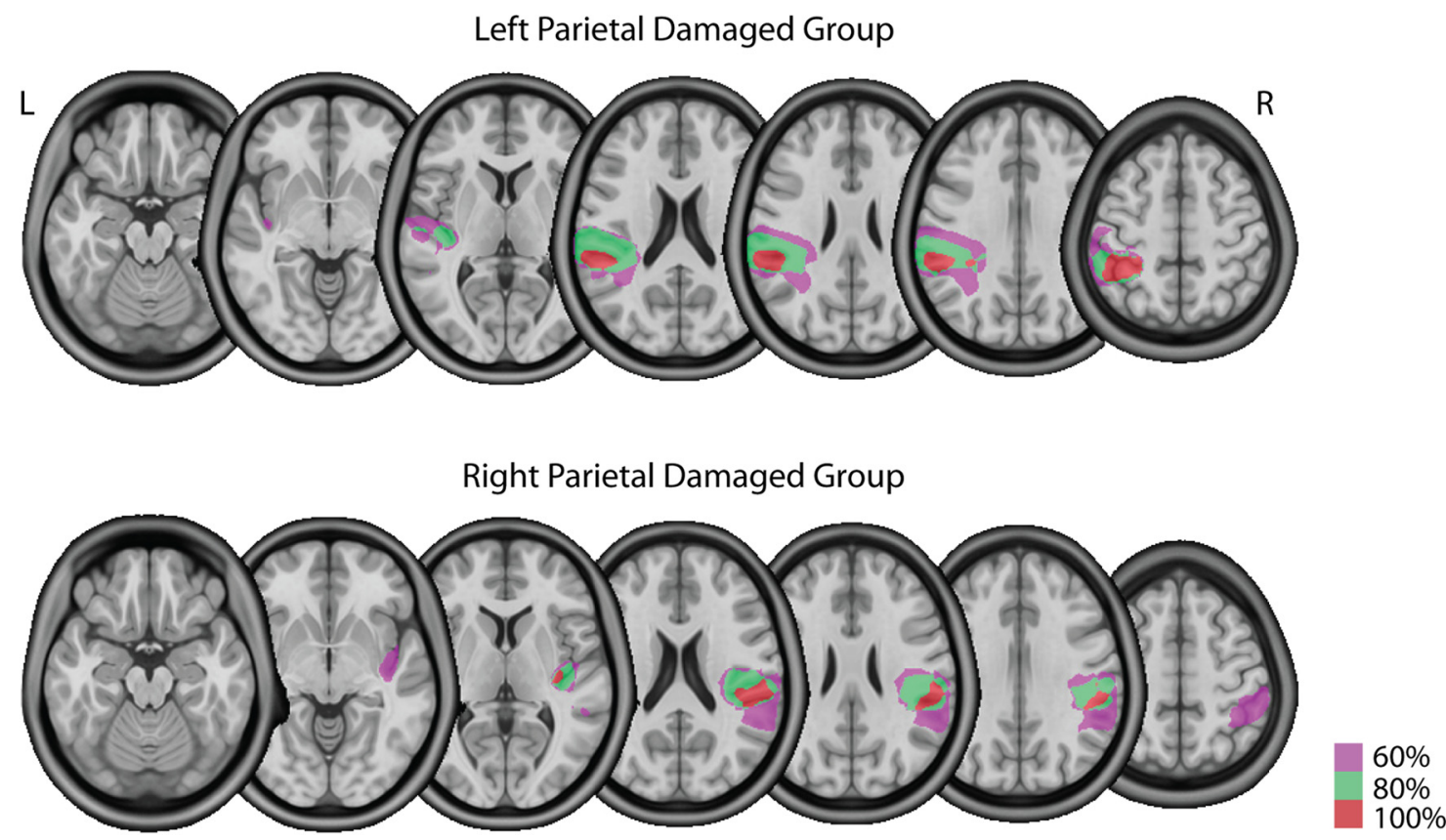

Figure 1. Overlap images showing the location of lesions in at least 60 (magenta), 80 (green), and 100 (red) percent of patients the left and right parietal damaged groups. Lesions were primarily in regions of posterior parietal cortex.

processing as required for adaptation (Krakauer et al., 2004), whereas left parietal activation might be associated with corrections for visuomotor errors (Desmurget et al., 1999) and planning visually targeted movements (Mutha et al., 2010). -

Such mixed neuroimaging findings are not only difficult to interpret, but they are also unable to resolve whether the activated parietal regions are necessary for adaptation. Lesion studies offer a potential solution to this problem. However, studies in patients with lesions restricted to parietal cortex are rare. One case report of a patient with bilateral parietal lesions showed that such damage prevents adaptation (Newport et al., 2006). Thus, while previous imaging and bilateral lesion studies have clearly demonstrated parietal involvement during visuomotor adaptation, they have not been able to distinguish whether left, right, or both parietal regions mediate adaptive behavior. We now address this issue by investigating visuomotor adaptation in patients with focal unilateral parietal damage.

\section{Materials and Methods}

Subjects. We tested 10 stroke patients [ 4 males; 5 left parietal damaged (LPD) and 5 right parietal damaged (RPD)] who performed with their contralesional affected arm and 14 healthy normal control participants [10 males; 7 left normal controls (LNCs) and 7 right normal controls (RNCs) who performed with their right or left arm, respectively, i.e., with the arm contralateral to their group designation]. All patients were in the chronic phase of injury ( $>6$ months after stroke). None of these patients were part of our previous study examining the dissociation of trajectory and position control mechanisms using the visuomotor rotation paradigm (Schaefer et al., 2009). All subjects gave informed consent before participation. The study was approved by the institutional review board of the New Mexico Veterans Affairs Healthcare System at the University of New Mexico (Albuquerque, NM). All control subjects were righthanded and all stroke subjects were right-handed before their stroke. Handedness was determined using the 10 item version of the Edinburgh inventory (Oldfield, 1971). Subjects were screened and excluded if they had a history of substance abuse, severe psychiatric diagnoses, peripheral movement restrictions from neuropathy or orthopedic injuries, nonstroke-related neurological problems for the stroke patients, or any neurological diagnoses for the healthy control group. All subjects were tested for language comprehension (Kertesz, 1982), and task performance indicated that subjects understood the task requirements without any difficulty. All subjects were also given a standardized test for limb apraxia (Haaland and Flaherty, 1984). A modified line cancellation test was administered to all subjects to test for visual neglect (Albert, 1973). Patients with two or more errors (of 21 possible) in the contralesional hemispace were classified as having visual neglect based on the fact that none of the control subjects made more than one error in either the left or the right hemispace. The Fugl-Meyer test of motor function (Fugl-Meyer et al., 1975) was given to the stroke patients as an indicator of hemiparesis.

High resolution T1-weighted magnetic resonance images were obtained in stroke patients that were then normalized to a standard template in Montreal Neurological Institute (MNI) space using unified segmentation and normalization routines in SPM8 (Ashburner and Friston, 2005) and custom MATLAB scripts. Lesions were then reconstructed on the anatomical images in Adobe Photoshop (Adobe Systems), and the traced lesions were converted back into volumes using custom MATLAB code. Volumes from multiple patients within a group (LPD or RPD) were then overlaid in MRICron (Rorden and Brett, 2000) to create overlap images showing areas of damage common to all patients within a group. These images are shown in Figure 1 , which confirms that lesions in our stroke patients were largely in the left or right parietal regions.

Experimental setup and task. Our experimental setup is shown in Figure $2 \mathrm{~A}$. Subjects sat facing a table with their palms facing downward and hands supported over the table using an air sled system to eliminate the effects of gravity and minimize friction. A cursor representing the position of the index finger tip, a start circle, and targets were projected using a horizontally mounted high-definition television (HDTV; Samsung Electronics) onto a mirror placed beneath it. The mirror blocked direct vision of the subjects arm but reflected the visual display to give the illusion that the display was in the same horizontal plane as the fingertip. Subjects performed reaching movements below the mirror. Position and orientation of the forearm and upper-arm segments were sampled using a Flock of Birds (FOB) system (Ascension Technology). The positions of the index finger tip, the lateral epicondyle of the humerus, and the acromion, directly posterior to the acromio-clavicular joint, were recorded 
using a stylus that was rigidly attached to a six degrees of freedom FOB sensor. This sensor was then attached to the upper arm segment, while another sensor was attached to the hand using a fitted strap. As sensor data were received, the three-dimensional position of the above-mentioned landmarks was computed using custom software, with the $X-Y$ plane parallel to the tabletop. We used our computed $X-Y$ coordinates of the fingertip to define the projected cursor position. The displayed cursor was the only visual feedback available to the subjects during the experiment. Digital data were collected at $130 \mathrm{~Hz}$. Custom algorithms for experimental control and data analysis were written in REAL BASIC (REAL Software) and Igor Pro (WaveMetrics), respectively.

The experimental task is shown in Figure $2 B$. Stroke subjects performed the task with their contralesional arm (i.e., right arm for LPDs and left arm for RPDs), while control subjects were randomly assigned to perform the task with either their left or right arm. The task itself consisted of reaching movements from a single start position to 8 targets at a radial distance of $12 \mathrm{~cm}$, arranged along the circumference of a circle $45^{\circ}$ apart. On any given trial only one of the targets was pseudo-randomly selected to be displayed on the screen, such that no target appeared consecutively. To initiate a trial, subjects brought the cursor (which represented their index finger) into the start circle. After a brief delay, the target for that trial appeared on the screen along with an audio-visual "go" signal, upon which subjects were required to reach to the target. Velocity feedback was provided in the form of a thermometer-type display, and subjects were encouraged to attain a peak speed of at least $0.5 \mathrm{~m} / \mathrm{s}$. Points were given if this speed requirement was satisfied and were based on final position accuracy relative to the center of the target.

Each subject performed three sessions of movements. Feedback about hand position by means of the screen cursor was given during all trials of every session. The first session of "baseline" trials had 120 trials, during which cursor and hand displacements were veridical. This session was used to establish baseline measures of performance. During the second "adaptation" session, the position of the cursor was rotated counterclockwise by $30^{\circ}$ relative to the start circle. This session included 208 trials and was used to examine adaptation to novel visuomotor conditions. The third session consisted of 120 trials, during which the cursor and hand motion were again veridical. This session was used to examine the presence of after-effects upon removal of the visuomotor rotation.

Data analysis. Finger, elbow, and shoulder positions were calculated from FOB sensor position and orientation data. These were then used to calculate shoulder and elbow joint angles. All kinematic data were lowpass filtered at $8 \mathrm{~Hz}$ (third-order, dual-pass Butterworth filter), and angular data were differentiated to yield velocity and acceleration values. Movement start was determined by identifying the time of peak velocity and searching backward in time for the first minimum in velocity below $3 \%$ of peak tangential velocity. Movement end was similarly determined by searching forward in time from peak velocity to find the first minimum in velocity below $3 \%$ of peak tangential velocity. Adaptation was quantified in terms of changes in direction errors at peak acceleration (initial direction error) and peak velocity. These errors were measured as the angular difference between the line joining the center of the start circle and finger position at either peak acceleration or peak velocity and the line from the center of the start circle to the target (Schaefer et al., matched again.

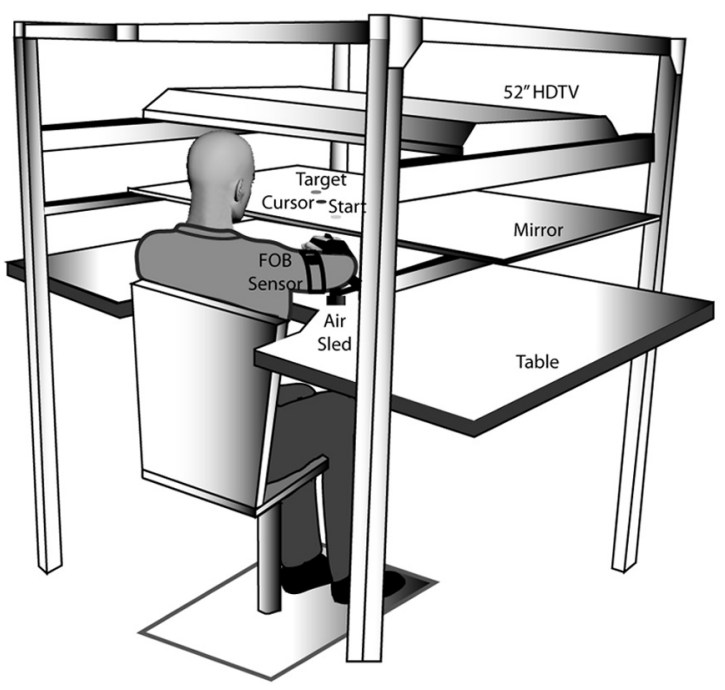

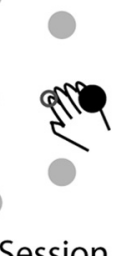

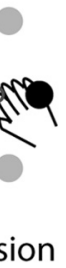

)
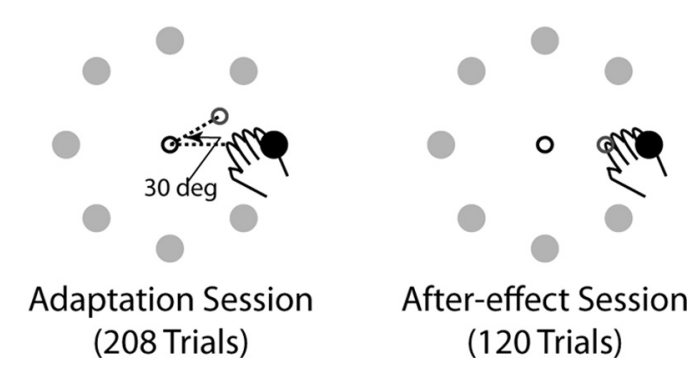

Figure 2. Experimental setup and task. $\boldsymbol{A}$, Subjects sat facing a mirror onto which a start position, targets, and a cursor representing the hand position were projected using a horizontally mounted HDTV and rested their arms in an air sled system 作 列 "session, the hand and cursor feedback were matched. During the "adaptation" session, the cursor was rotated $30^{\circ}$ (deg) counterclockwise relative to the direction of hand motion, while during the "after-effect" session the hand and cursor were

2009; Mutha et al., 2010). We focus on these two times in movement because patterns up to the time of peak acceleration have been shown previously to be resistant to changes in sensory feedback and thus reflect preplanned or predictive components of movement (Gordon and Ghez, 1987; Sainburg and Schaefer, 2004), while the measure at peak velocity reflects the stability of movement direction through roughly the first half of movement. Direction errors were calculated in a right hand coordinate system, with negative values of this measure indicating that hand paths were directed medial (counterclockwise) to the target line.

For analysis purposes we considered each session as cycles of movements. A cycle was defined as a complete series of movements to each of the eight targets. The baseline, rotation, and after-effects sessions thus included 15, 26, and 15 cycles, respectively. Our primary interest was in examining the change in initial direction error from the first to the last cycle of the rotation session for each group. We also compared the extent of adaptation in each group by comparing the after-effects (initial direction error) during the first cycle of the third session.

Statistical analyses were conducted using repeated-measures ANOVA with group (LNC, RNC, LPD, RPD) as the between-subject factor and cycle (first or last) as the within-subject factor. Tukey's post hoc tests were conducted when warranted by significant main effects and interactions.

\section{Results}

\section{Subject characteristics}

Table 1 shows the age, education, and handedness for all participants and the score on the upper-extremity motor component of the Fugl-Meyer assessment for the LPD and RPD stroke subjects. Our groups were not significantly different in age $\left(F_{(3,20)}=\right.$ 
Table 1. Participant demographics including age, education, and handedness for all subjects and upper-extremity Fugl-Meyer scores for the LPD and RPD patients

\begin{tabular}{|c|c|c|c|c|}
\hline Subject & Age (years) & Education (years) & Handedness $^{a}$ & Upper Extremity Fugl-Meyer Score \\
\hline LNC1 & 66 & 14 & $\mathrm{R}$ & \\
\hline LNC2 & 64 & 18 & $\mathrm{R}$ & \\
\hline LNC3 & 54 & 16 & $\mathrm{R}$ & \\
\hline LNC4 & 53 & 14 & $\mathrm{R}$ & \\
\hline LNC5 & 50 & 16 & $\mathrm{R}$ & \\
\hline LNC6 & 73 & 13 & $\mathrm{R}$ & \\
\hline LNC7 & 55 & 12 & $\mathrm{R}$ & \\
\hline LPD1 & 73 & 16 & $\mathrm{R}$ & 66 \\
\hline LPD2 & 66 & 13 & $\mathrm{R}$ & 61 \\
\hline LPD3 & 83 & 18 & $\mathrm{R}$ & 64 \\
\hline LPD4 & 61 & 16 & $\mathrm{R}$ & 50 \\
\hline LPD5 & 71 & 18 & $\mathrm{R}$ & 65 \\
\hline RNC1 & 62 & 18 & $\mathrm{R}$ & \\
\hline RNC2 & 62 & 12 & $\mathrm{R}$ & \\
\hline RNC3 & 65 & 12 & $\mathrm{R}$ & \\
\hline RNC4 & 78 & 16 & $\mathrm{R}$ & \\
\hline RNC5 & 48 & 17 & $\mathrm{R}$ & \\
\hline RNC6 & 67 & 20 & $\mathrm{R}$ & \\
\hline RNC7 & 54 & 18 & $\mathrm{R}$ & \\
\hline RPD1 & 61 & 12 & $\mathrm{R}$ & 59 \\
\hline RPD2 & 56 & 12 & $\mathrm{R}$ & 66 \\
\hline RPD3 & 78 & 18 & $\mathrm{R}$ & 66 \\
\hline RPD4 & 58 & 11 & $\mathrm{R}$ & 55 \\
\hline RPD5 & 42 & 12 & $\mathrm{R}$ & 62 \\
\hline
\end{tabular}

${ }^{a}$ Handedness in stroke patients refers to their handedness before stroke. R, Right.

$1.6664, p=0.2062)$ or education $\left(F_{(3,20)}=1.8689, p=0.1674\right)$. None of the patients in the LPD or RPD groups were classified as having spatial neglect. Consistent with several previous reports (Haaland et al., 2000; for review, see Goldenberg, 2009), limb apraxia was more common after left rather than right parietal damage. None of the RPD patients were apraxic, while four of five LPD patients were classified as having limb apraxia. The FuglMeyer test was administered to the stroke patients because we tested their contralesional affected arm and we wanted to ensure that hemiparesis would not impact their motor performance. The mean score on the Fugl-Meyer for the LPD group was 61.2 $( \pm 2.92 \mathrm{SE})$ and for the RPD group it was $61.6( \pm 2.11 \mathrm{SE})$ out of a maximum possible 66 , and there was no statistical difference between the two stroke groups $(p=0.9147)$. These scores indicated that our patients were very mildly hemiparetic, and the comparable scores in the LPD and RPD groups indicated that the differences in task performance in these groups were not attributable to differences in the degree of hemiparesis. Furthermore, arm proprioception, measured as a component of the FuglMeyer assessment, was intact in all patients. Lesion volume was also not significantly different between the two patient groups $(p=0.8704)$. Mean lesion volume in the LPD group was $87.16 \mathrm{cc}$ ( $\mathrm{SE} \pm 18.63 \mathrm{cc}$ ), while the mean lesion volume in the RPD group was $81.75 \mathrm{cc}$ ( $\mathrm{SE} \pm 25.95 \mathrm{cc})$. Again, our match for lesion volume precluded the possibility that any difference in task performance could be attributed to differences in lesion volume between the LPD and RPD groups.

\section{Task performance}

In general, stroke subjects tended to move more slowly than the control participants regardless of the experimental session, as shown in the bar plots comparing peak velocity across groups and sessions in Figure $3 A-C$. This pattern was confirmed by a significant main effect of group in our $\operatorname{ANOVA}\left(F_{(3,20)}=4.2192, p=\right.$ 0.0183 ). This is consistent with previous studies that have dem- onstrated a disturbance in contralesional arm movement speed following stroke (Levin, 1996; Beer et al., 2000). Importantly however, there were no significant differences in peak velocity between the LPD and RPD stroke groups in the baseline ( $p=$ $0.8471)$, adaptation $(p=0.8643)$, or after-effect sessions ( $p=$ $0.9850)$. Because of the lack of significant peak velocity differences between the two stroke groups, we do not to emphasize these findings further. Rather, we focus on the more critical group differences in adaptation to the imposed visuomotor rotation, quantified through changes in movement direction during the early phases of the movement.

\section{Baseline performance}

During the baseline session, visual feedback of hand location (cursor) was matched to the actual hand location. Figure $3 D$ shows the last cycle of baseline movements to the eight targets for representative subjects from each of our four groups (LNC, LPD, $\mathrm{RNC}$, and RPD). The velocity profiles for these eight movements are also shown in the inset of each hand path plot. As can be seen from Figure $3 D$, for all four representative subjects, these movements were initially directed toward the targets and they ended accurately. In general, the velocity profiles associated with these baseline movements were fairly smooth and bell-shaped for all subjects. The LPD patient showed double-peaked velocity profiles on a few trials of this baseline cycle. However, these secondary peaks were small and occurred late in the movement and were likely associated with adjustments in movement trajectory for accurately stopping on the target. Across all subjects, we did not find any group differences for initial direction error $\left(F_{(3,20)}=\right.$ $1.6549, p=0.2086)$ or final position accuracy $\left(F_{(3,20)}=0.8817\right.$, $p=0.4673)$ during the baseline trials, suggesting that baseline performance was consistent across all four groups.

\section{Adaptation}

Figure $3 E$ shows the pattern of movements and the associated velocity profiles for the same four representative subjects when they were first exposed to the $30^{\circ}$ visuomotor rotation (first cycle of movements to the eight targets). As expected, movements were initially directed similar to baseline trials. However, because the cursor deviation introduced an error between the visually displayed cursor and the actual hand positions, subjects altered their movement trajectory to bring the cursor toward the target. This resulted in highly curved hand paths upon initial exposure to the rotation. The pattern of the velocity profiles also reflected the trajectory modifications and was fairly similar across all participants. As shown in the insets of Figure $3 E$, velocity profiles were multipeaked in all representative subjects for these trials, with the secondary components reflecting the corrective response to the imposed cursor deviation. Figure $4 A, C$ show the consistency of this pattern in the hand paths and velocity profiles in all remaining patients from the LPD and RPD groups, respectively.

Across all subjects, the magnitude of the initial direction error during the first cycle of the adaptation session was close to $30^{\circ}$ (Fig. 5A), similar to the magnitude of the imposed rotation. This error magnitude did not differ significantly across the four groups $\left(F_{(3,20)}=0.5414, p=0.6595\right)$ indicating that performance of all four groups was similarly affected by initial exposure to the rotation.

Over the course of the adaptation session, the control subjects (LNC, RNC) and right parietal damaged patients improved their initial movement direction. This is evident in Figure $3 F$ for our representative subjects as well as in Figure $4 D$, which shows the hand paths for the remaining four subjects in the RPD group 
A

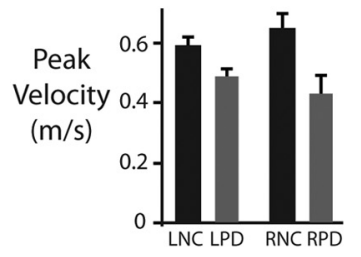

D

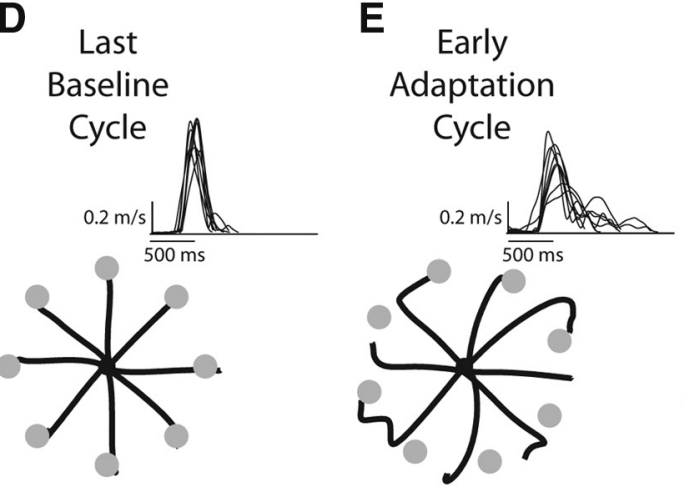

LNC
B

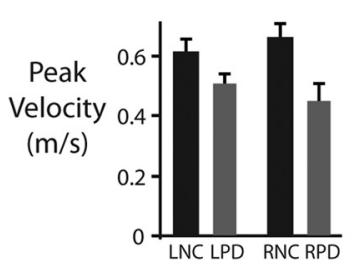

C

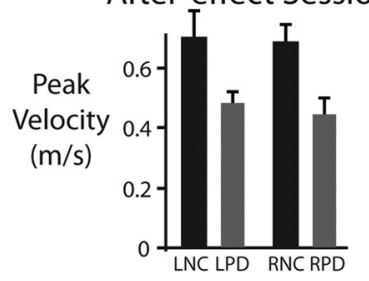

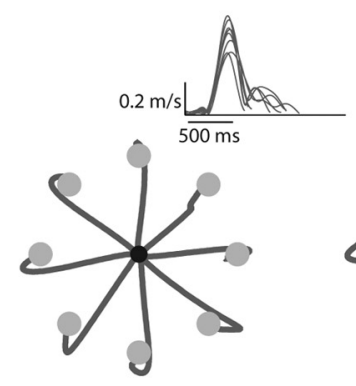

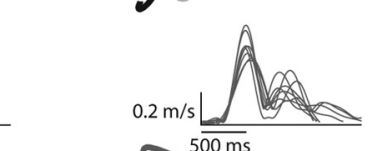

F

Late

Adaptation

Cycle
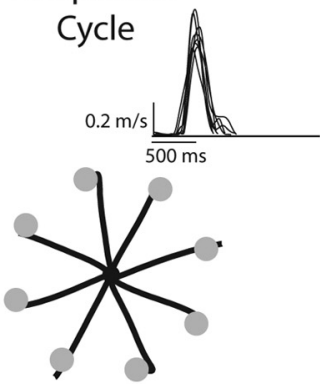

G First

After-effect

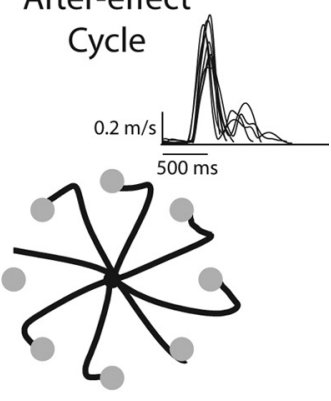

LPD 1
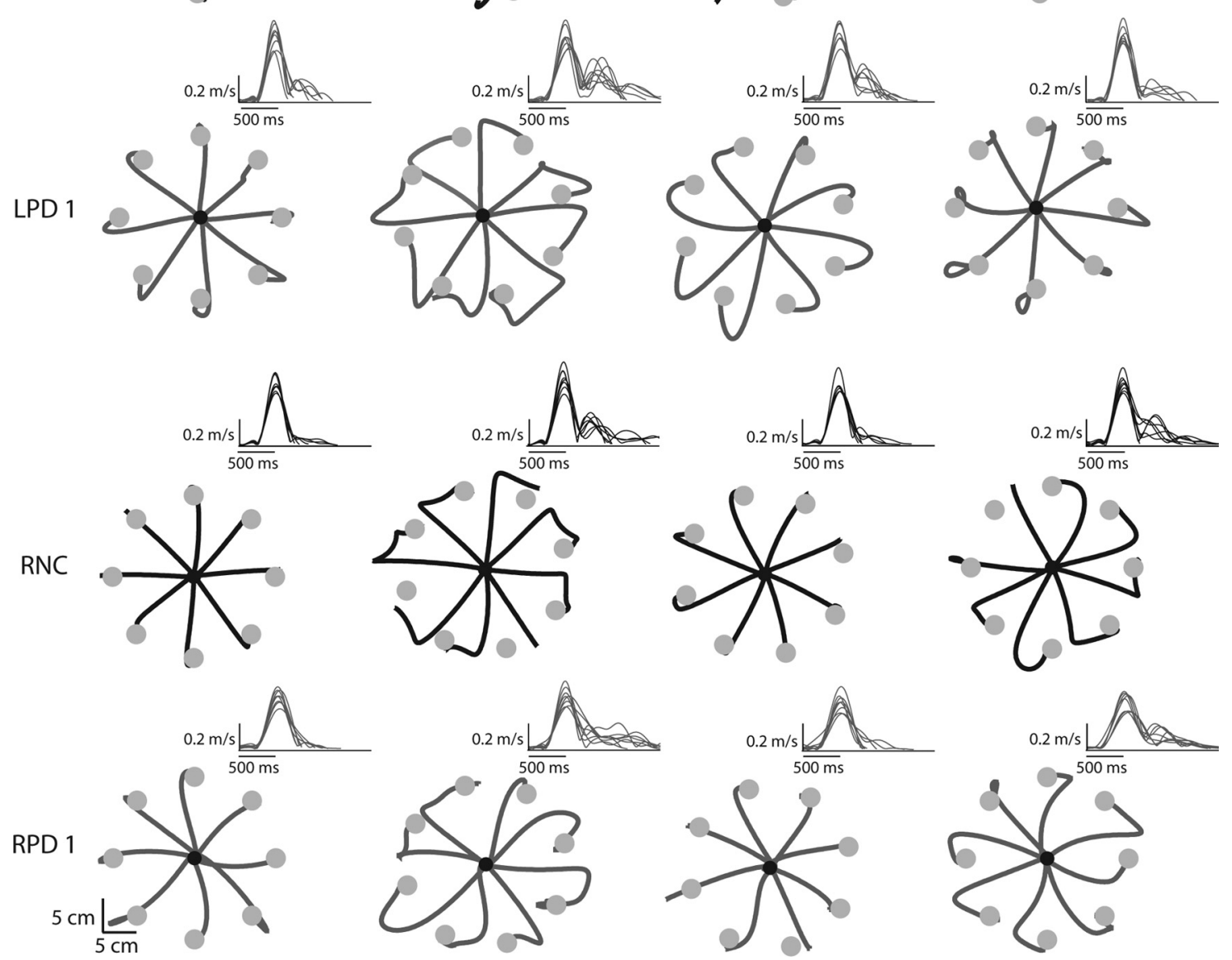

Figure 3. Comparison of peak velocities and movement profiles. $A$, Mean \pm SE peak velocities across all subjects in the left and right normal control (LNC, RNC, black bars) and left and right parietal damaged (LPD, RPD, gray bars) groups during the baseline session. $B$, Mean \pm SE peak velocities across all subjects in the four groups during the adaptation session. $C$, Mean \pm SE. peak velocities across all subjects in the four groups during the after-effect session. $\boldsymbol{D}$, Hand paths for representative subjects in the LNC and RNC groups (black lines) and the LPD and RPD groups (gray lines) during the last eight trials (last cycle) of the baseline session. $\boldsymbol{E}$, First cycle of the adaptation session. $\boldsymbol{F}$, Last cycle of the adaptation session. $\boldsymbol{G}$, First cycle of the after-effects session. Insets for the hand paths in $\boldsymbol{D}-\boldsymbol{G}$ show the velocity profiles associated with those movements. 

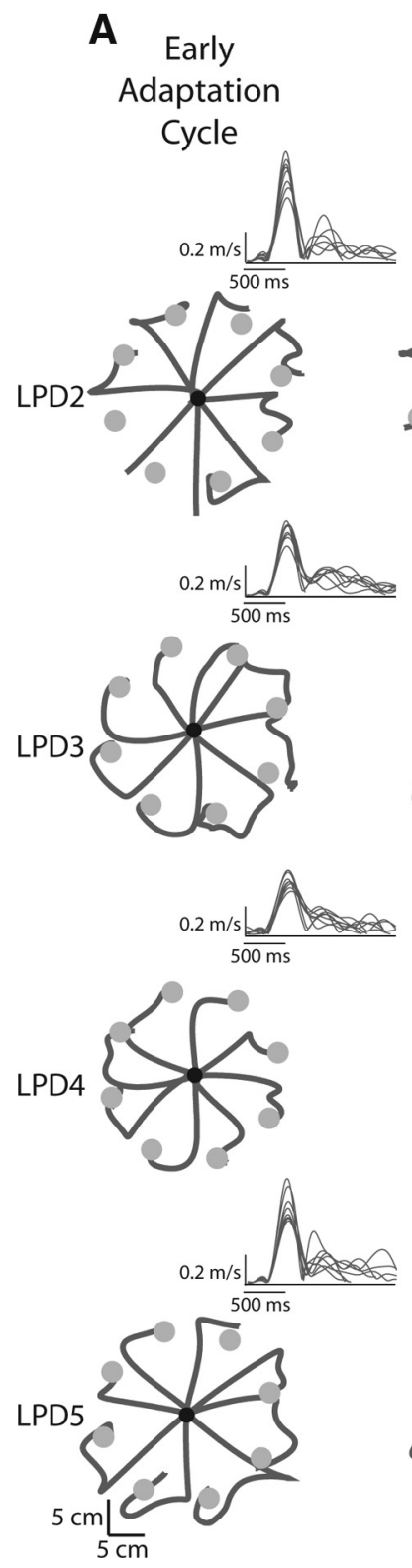

B Late
Adaptation
Cycle

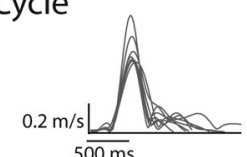

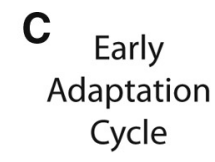
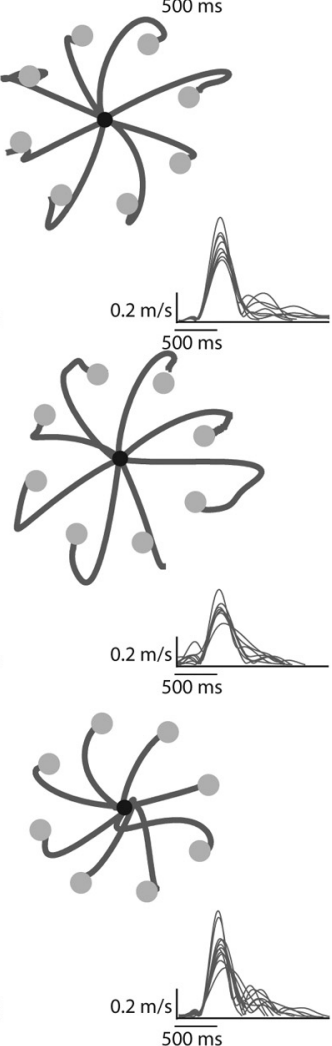

RPD2
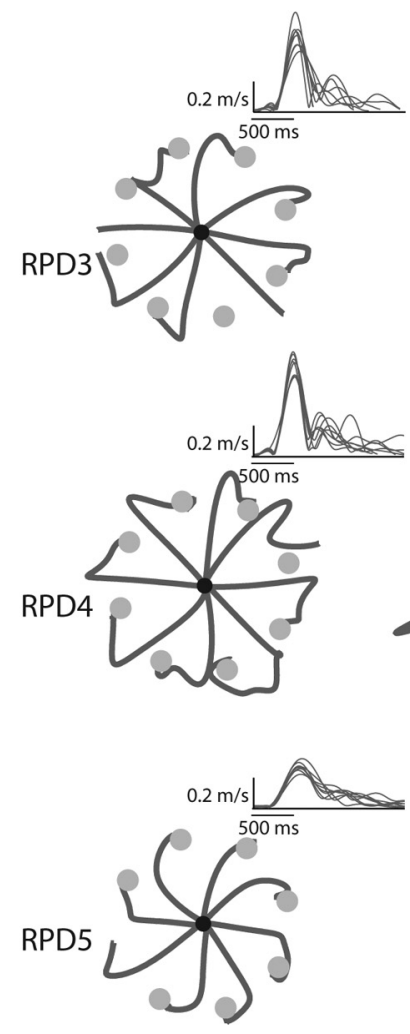
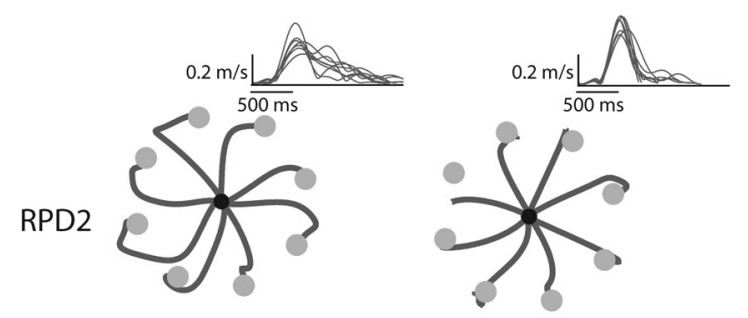

\section{Late \\ Adaptation Cycle}
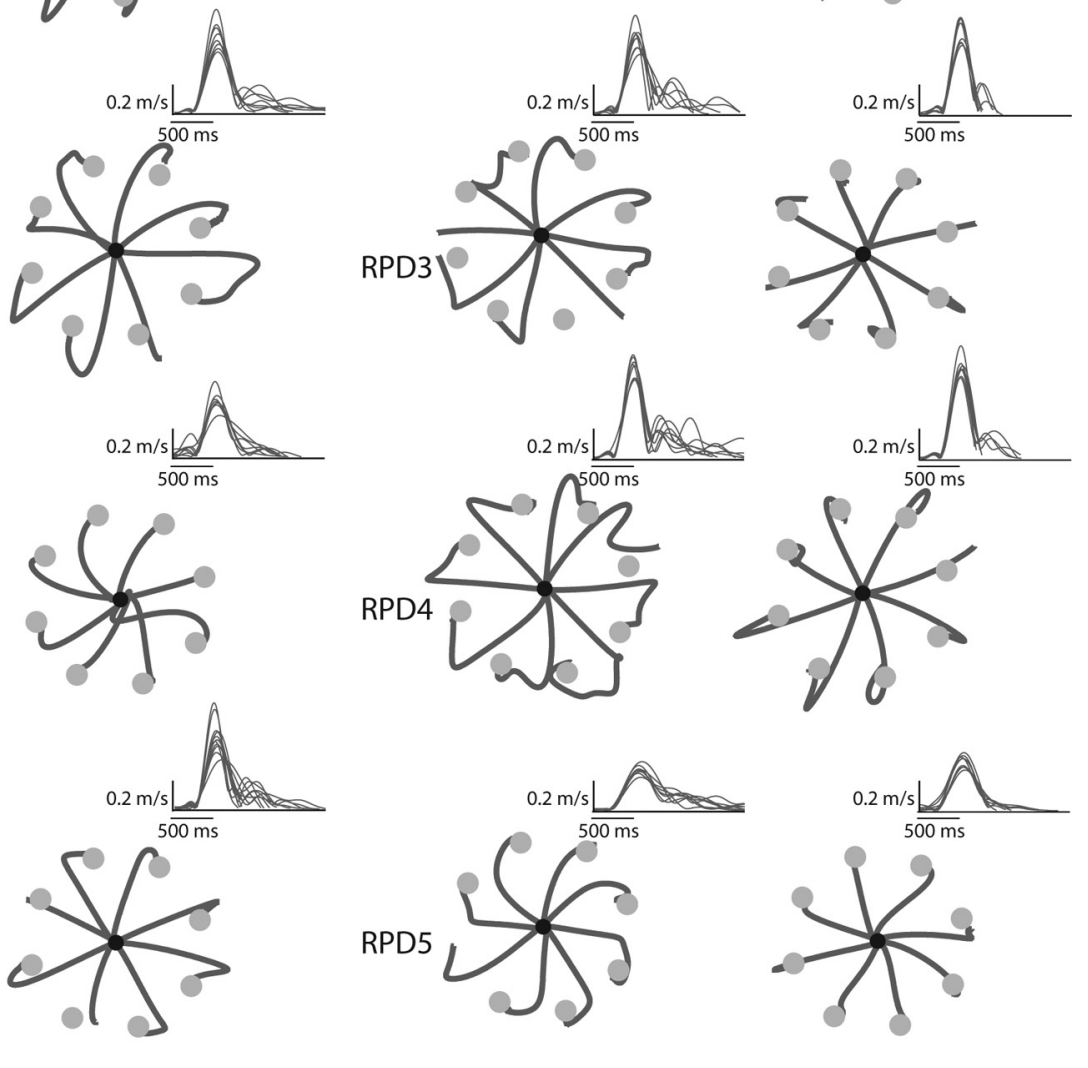

Figure 4. Hand paths for the remaining four subjects in each of the left and right parietal damaged (LPD, RPD) groups. $A$, First cycle of the adaptation session for the LPD subjects. $B$, Last cycle of the adaptation session for the LPD subjects. $\boldsymbol{C}$, First cycle of the adaptation session for the RPD subjects. $\boldsymbol{D}$, Last cycle of the adaptation session for the RPD subjects. Insets for the hand paths in $\boldsymbol{A}-\boldsymbol{D}$ show the velocity profiles associated with those movements.

during the last cycle of the adaptation session. These movements were remarkably straight and were directed toward the (rotated) target, which ensured that the cursor headed directly toward the visually displayed target. The velocity profiles associated with these movements became smoother and largely single-peaked, with only a few trials still showing a prominent second peak that could still be clearly attributed to a response correcting for directional deviations (rather than movement reversal, which occurred, for example, for subject RPD4 in Fig. $4 D$ ). Figure $5 A$ shows the initial direction error across all subjects in each group for every cycle of the adaptation session. This plot shows the consistent improvement in initial movement direction across all subjects in these three groups, with errors close to about $5^{\circ}$ in the last cycle.

In contrast, the left parietal damaged patients showed a striking deficit in adaptation of initial movement direction. Even after experiencing the rotation for over 200 trials, these subjects showed little to no adaptation in initial direction. The representative LPD subject shown in Figure $3 C$ continued to initiate movements in the baseline target direction and relied on correcting them online to bring the cursor toward the target even during the last cycle of the adaptation session. The remaining subjects in this group, shown in Figure 4B, also demonstrated a similar pattern. The velocity profiles associated with these movements continued to show multiple peaks related to online movement corrections. This pattern of large initial direction errors was consistent across the LPD patients as a group (Fig. 5A), who continued to show errors that were on average $\sim 23$ degrees $\left(\mathrm{SE} \pm 2^{\circ}\right.$ ) during the last cycle of adaptation. A significant group (LNC, RNC, LPD, RPD) $\times$ cycle (first adaptation, last adaptation) interaction $\left(F_{(3,20)}=4.6667, p=0.0125\right)$ confirmed this pattern of results statistically. Post hoc analysis revealed that while initial direction error was similar across all four groups during the first cycle, the LPD group showed significantly larger direction errors 
A

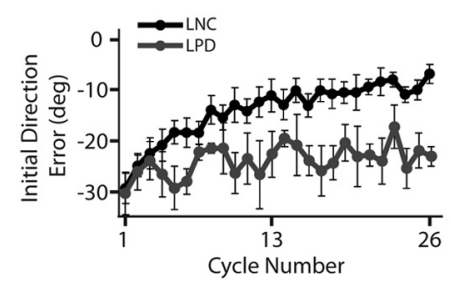

\section{Adaptation Session}

\section{B}

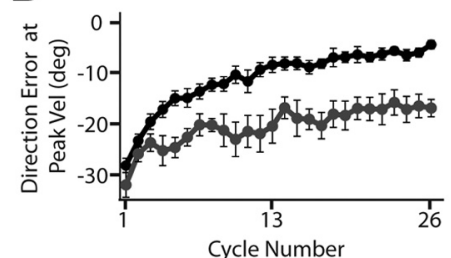

Cycle Number

C
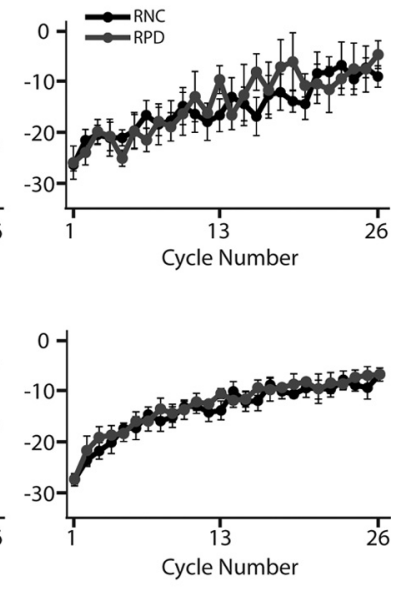

After-effect

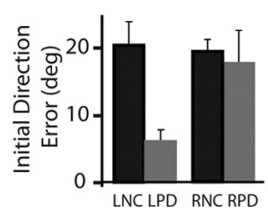

Figure 5. Lack of adaptation and after-effects in the left parietal damaged group. $\boldsymbol{A}$, Mean $\pm S E$ initial direction error (direction error at peak acceleration) across all cycles of the adaptation session. $\boldsymbol{B}$, Mean \pm SE direction error at peak velocity across all cycles of the adaptation session. Data for the left control, LNC, and left parietal damaged, LPD, groups are shown in the left panel, while those for the right control, RNC, and right parietal damaged, RPD, groups are shown in the right panel. Control groups are shown using black lines, while stroke groups are shown using gray lines. C, Mean \pm SE initial direction error during the first cycle of the aftereffect session for the LNC and RNC (black) and LPD and RPD (gray) groups.

relative to all the other three groups ( $p<0.0092$ in all cases) on the last cycle. Importantly, Figure $5 A$ shows that the deficit in initial direction was not associated with an initial improvement and a later degradation of performance, but was rather sustained throughout the adaptation session. Thus, LPD patients showed significant deficits in adapting their initial direction to the imposed visuomotor rotation.

The direction error at peak velocity provides an index of the stability of initial direction errors through a longer initial phase of motion. Figure $5 B$ shows the direction error at peak velocity across all subjects in the four groups. Consistent with the pattern observed for initial direction error, the LNC, RNC, and RPD groups showed clear improvement in this measure over the course of the adaptation session. In contrast, the LPD group showed some improvement initially, but its performance showed an asymptote after the first few cycles of experiencing the rotation. This was statistically confirmed by a significant group (LNC, RNC, LPD, RPD) $\times$ cycle (first adaptation, last adaptation) interaction effect $\left(F_{(3,20)}=9.6372, p=0.0004\right)$, with post hoc tests showing significantly larger direction errors at peak velocity for the LPD group relative to all other groups on the last cycle of adaptation ( $p<0.0001$ in all cases). Thus, through the time of peak velocity, the LPD patients continued to demonstrate larger direction errors relative to all other groups.

After-effects

Besides assessing performance in the last cycle of the adaptation session, an important test of whether the visuomotor rotation has been learned is to test for after-effects following the adaptation session. Under conditions of veridical hand and cursor motion following adaptation, healthy subjects typically show movement trajectories directed opposite to the direction of the previously imposed rotation. Our data reveal that this was indeed the case for the LNC, RNC, and RPD subjects, but not the LPD patients. Figure $3 G$ shows the first cycle of the after-effect session for the same representative subjects. As can be seen from this figure, movements in the first cycle of the after-effect session were directed clockwise relative to the intended target, opposite to the imposed counterclockwise rotation in the previous session, but only for the LNC, RNC, and RPD subjects. The velocity profiles associated with these movements in these subjects showed multiple peaks, with the secondary components associated with the directional corrections to bring the cursor to the displayed target. The LPD participant, however, performed movements that were aimed fairly accurately toward the intended target, thus demonstrating a negligible after-effect. The velocity profiles for this LPD subject appeared similar to the baseline velocity profiles with small secondary peaks that occurred late and only on a few trials, possibly related to accurately stopping on the target rather than a large response correcting for movement direction. The negligible after-effect was consistent across all LPD subjects as shown in Figure $5 C$ and as evidenced by a significant main effect of group $\left(F_{(3,20)}=3.526, p=0.0212\right)$ for initial direction error during the first after-effect cycle. Post hoc analysis showed smaller aftereffects for the LPD group relative to the control participants $(p<$ $0.05)$. For the LNC, RNC, and RPD groups the magnitude of the after-effect was similar ( $p>0.90$ in all cases). Thus, the lack of after-effects in the LPD group confirms that this group did not learn the novel visuomotor rotation.

\section{Subanalysis}

Our lesion data showed that while all of our LPD patients had damage in superior parietal regions, $60 \%$ of our RPD group had lesions in this area. To rule out the possibility that the differences in the LPD and RPD groups were due to greater superior parietal involvement in the LPD group, we performed a subanalysis comparing the performance of the five patients with superior parietal damage in the LPD group to the three patients with such damage in the RPD group.

The pattern of results for the subanalyses was similar to the analyses done with all subjects included. More specifically, there was a significant group $\left(\mathrm{LNC}, \mathrm{RNC}, \mathrm{LPD}, \mathrm{RPD}_{\text {subgroup }}\right) \times$ cycle (first, last) interaction $\left(F_{(3,18)}=5.2155, p=0.0091\right)$ for initial direction error during the adaptation session. Post hoc analysis indicated that while initial direction error was similar across all groups during the first cycle of exposure to the rotation $(p>0.94$ in all cases), only the LPD patients continued to show large direction errors during the last cycle of the adaptation session $(p<$ 0.0099 in all cases). These results were similar to those obtained with our larger groups. The pattern of results for the after-effect session was also similar. We observed a significant effect of group $\left(F_{(3,18)}=4.3842, p=0.0175\right)$ on initial direction error during the first cycle of the after-effect session, with post hoc tests revealing no differences in the magnitude of the after-effect for the RNC, LNC, and RPD groups ( $p>0.99$ in all cases), but a significantly smaller after-effect in the LPD patients relative to the control group $(p<0.05)$. The similar pattern of results when including the full sample or only patients with superior parietal damage confirmed that the slight difference in the number of patients with superior parietal damage in the two groups did not determine our overall pattern of results. 


\section{Discussion}

We examined the effect of focal left or right parietal damage on adaptive behavior. To our knowledge, this is the first study of visuomotor adaptation in a group of patients with damage to left or right parietal regions. Patients with left parietal damage failed to adapt their movement direction in response to a visuomotor rotation and showed no after-effects upon its removal. In contrast, patients with right parietal damage adapted their movement direction normally and showed large after-effects. Thus, our data indicate that left but not right parietal regions are critical for visuomotor adaptation.

\section{Role of left parietal circuits in adaptive visuomotor control}

While previous studies have demonstrated involvement of parietal regions during adaptation, they have been equivocal about whether left, right, or both parietal cortices are important for this process. Early positron emission tomography imaging studies (Clower et al., 1996) showed that parietal regions contralateral to the arm performing a prism adaptation task showed increased blood flow, particularly around the intraparietal sulcus. However, using functional magnetic resonance imaging, other studies have shown bilateral parietal activation during visuomotor learning, with somewhat larger responses in left parietal areas. For instance, Grefkes et al. (2004) showed activation of the intraparietal sulcus region bilaterally, with larger activation in the left hemisphere. Graydon et al. (2005) also showed bilateral inferior parietal activation, with additional activation of the left superior parietal lobe. Further, Diedrichsen et al. (2005) showed activation in anterior parietal regions [Brodmann area (BA) 5] bilaterally, again with slightly stronger activation in the left hemisphere. Thus, while the localization of activity within parietal regions was different across these studies, they all emphasized bilateral parietal involvement during visuomotor adaptation. In contrast, studies by Ghilardi et al. showed activation of right posterior parietal cortex, particularly BA 7, during rotation learning (Ghilardi et al., 2000; Krakauer et al., 2004). However, more recent results by Danckert et al. (2008) showed modulation of activity in anterior intraparietal sulcus regions of only the left hemisphere during adaptation. Thus, while informative, findings from these imaging studies are somewhat inconsistent, and they also do not resolve the question of whether the activated left, right, or bilateral parietal regions are actually critical for adaptation. Lesion studies may potentially answer this question, but unfortunately experiments examining the impact of damage to parietal regions on visuomotor adaptation are rare. One case study of a patient with bilateral parietal damage demonstrated poor adaptation (Newport et al., 2006), but another case report in a patient with bilateral optic ataxia, a disorder that usually occurs following bilateral superior parietal damage, concluded that adaptation was intact (Pisella et al., 2004). Thus, collectively, the results from imaging and lesion studies have been quite controversial. Our results clearly illustrate that adaptive visuomotor control critically depends on left parietal regions.

What specific role do these left parietal regions play in the adaptive control process? Some reports have suggested that during movement the actual visual and proprioceptive feedback is integrated with the predicted sensory consequences of motor commands, possibly generated via a forward model, to derive an estimate of the current state of the limb (Miall and Wolpert, 1996; Wolpert and Ghahramani, 2000; Shadmehr and Krakauer, 2008). Under novel visuomotor conditions, knowledge of the current state is used to correct the ongoing movement and to modify and restore our representations of the relationship between movement commands and their effects on limb and environmental state. Online corrections ensure accurate completion of the ongoing movement, while storage of the new visuomotor relationship, or remapping, leads to more accurate planning of subsequent movements. It is unclear whether the processes of state estimation and visuomotor remapping are mediated by distinct neural mechanisms. Some studies however suggest that both might depend on left parietal circuits. For example, Desmurget et al. (1999) demonstrated that transcranial magnetic stimulation over left parietal cortex impaired the ability to correct movements online in response to a shift in target location, presumably by disrupting processes involved in estimation of limb and target position and/or integration of these estimates. On the other hand, Wolpert et al. (1998) demonstrated that a patient with a cyst in the left parietal lobe was unable to maintain a target grip force over time when holding an object unless she was provided with visual feedback of the hand and object. This deficit was suggested to arise from an inability to store an updated map of limb state.

Our LPD patients were able to bring the cursor to the intended target (Figs. $3 E, F, 4 A, B$ ), suggesting an intact ability to derive and use information about the current limb state to correct their movements online. This suggests that state estimation may not be exclusively dependent on left parietal regions. More importantly however, our LPD patients did not improve initial direction over successive trials of the adaptation session, a process dependent on storage of the novel visuomotor relationship. This suggests that the ability to form and maintain internal representations of the relationship between movement commands and the state of the limb and the environment depends on left parietal circuits. In this regard, our results are consistent with the suggestion of Wolpert et al. (1998) and also agree with studies in patients with ideomotor limb apraxia that have shown spatiotemporal movement deficits thought to arise from damage to internal representations of skilled movement stored in left parietal regions (Haaland et al., 2000; Buxbaum et al., 2007; Goldenberg, 2009). Indeed, a larger number of LPD patients in the current study were apraxic.

It is possible that state estimates are derived in the cerebellum and then relayed to parietal regions that mediate visuomotor remapping. Miall and Wolpert (1996) have reviewed how cerebellar circuitry is suited for state estimation using a forward model. Furthermore, Tanaka et al. (2009) have recently developed a computational model in which state estimates derived from the cerebellum are relayed to parietal cortex, where visuomotor remapping occurs. While we cannot be absolutely certain that parietal regions are the locus of remapping, our results indicate that the ability to remap our representations depends critically on left parietal regions.

\section{Lateralization of parietal function}

Our findings indicate that LPD but not RPD patients initiated their movements in the wrong direction throughout the adaptation session, suggesting that their motor plan was not accurate. As discussed above, this deficit is most likely the consequence of an inability to form or modify internal representations following left parietal damage. This finding of lateralized mechanisms has important implications for understanding left and right hemisphere specialization for movement control and is of great significance for assessing the impact of unilateral brain damage on function. First, our data suggest that while right parietal regions may be 
activated during adaptation, these regions are not critical for the adaptation process. This result is in agreement with our recent work (Schaefer et al., 2009) in which patients with damage over a large part of their right hemisphere were able to adapt their movement trajectory when performing with their ipsilesional arm on a similar rotation-learning task. Intact adaptation in our RPD patients is also consistent with studies that have demonstrated adequate prism adaptation in patients with left hemispatial neglect, which occurs most commonly following right parietal damage (Rossetti et al., 1998; McIntosh et al., 2002; Pisella et al., 2002). In fact, prism adaptation has recently been evaluated as a rehabilitation tool for neglect in such patients (Turton et al., 2010). Second, the current findings extend recent results from our laboratory that have emphasized a left hemisphere specialization for predictive control of trajectory features of arm movements, such as initial movement direction (for review, see Sainburg, 2005). We previously (Schaefer et al., 2009) showed that patients with a range of left hemisphere lesions that included the parietal lobe did not adapt when performing with their ipsilesional arm. While it is likely that parietal lesions influenced this deficit, Schaefer et al. (2009) could not directly address the role of these regions during adaptation. By focusing on a group of patients with focal damage, our current results demonstrate that a critical node for such adaptation is the left parietal cortex. Third, an interesting possibility that the current results in combination with our previous findings (Schaefer et al., 2009) raise is that left parietal regions may be important for planning movements of either arm. This idea is consistent with the purported specialization of left parietal circuits for planning movement sequences of either arm (Haaland et al., 2004), motor attention (Rushworth et al., 1997, 2001), movement selection (Rushworth et al., 2003) and arm selection (Oliveira et al., 2010). This suggests that damage to left parietal circuits should impair adaptation when using the contralesional or ipsilesional arm, whereas in the case of right parietal damage, intact left parietal regions may yield relatively accurate movement planning for either arm. We are exploring this possibility further.

\section{References}

Albert ML (1973) A simple test of visual neglect. Neurology 23:658-664.

Ashburner J, Friston KJ (2005) Unified segmentation. Neuroimage 26:839-851.

Beer RF, Dewald JP, Rymer WZ (2000) Deficits in the coordination of multijoint arm movements in patients with hemiparesis: evidence for disturbed control of limb dynamics. Exp Brain Res 131:305-319.

Buxbaum LJ, Kyle K, Grossman M, Coslett HB (2007) Left inferior parietal representations for skilled hand-object interactions: evidence from stroke and corticobasal degeneration. Cortex 43:411-423.

Clower DM, Hoffman JM, Votaw JR, Faber TL, Woods RP, Alexander GE (1996) Role of posterior parietal cortex in the recalibration of visually guided reaching. Nature 383:618-621.

Danckert J, Ferber S, Goodale MA (2008) Direct effects of prismatic lenses on visuomotor control: an event-related functional MRI study. Eur J Neurosci 28:1696-1704.

Desmurget M, Epstein CM, Turner RS, Prablanc C, Alexander GE, Grafton ST (1999) Role of the posterior parietal cortex in updating reaching movements to a visual target. Nat Neurosci 2:563-567.

Diedrichsen J, Hashambhoy Y, Rane T, Shadmehr R (2005) Neural correlates of reach errors. J Neurosci 25:9919-9931.

Fugl-Meyer AR, Jääskö L, Leyman I, Olsson S, Steglind S (1975) The poststroke hemiplegic patient. 1. A method for evaluation of physical performance. Scand J Rehabil Med 7:13-31.

Gazzaniga MS (2000) Cerebral specialization and interhemispheric communication: does the corpus callosum enable the human condition? Brain 123:1293-1326.

Ghilardi M, Ghez C, Dhawan V, Moeller J, Mentis M, Nakamura T, Antonini
A, Eidelberg D (2000) Patterns of regional brain activation associated with different forms of motor learning. Brain Res 871:127-145.

Goldenberg G (2009) Apraxia and the parietal lobes. Neuropsychologia $47: 1449-1459$.

Gordon J, Ghez C (1987) Trajectory control in targeted force impulses. II. Pulse height control. Exp Brain Res 67:241-252.

Graydon FX, Friston KJ, Thomas CG, Brooks VB, Menon RS (2005) Learning-related fMRI activation associated with a rotational visuomotor transformation. Brain Res Cogn Brain Res 22:373-383.

Grefkes C, Ritzl A, Zilles K, Fink GR (2004) Human medial intraparietal cortex subserves visuomotor coordinate transformation. Neuroimage 23:1494-1506.

Haaland KY, Flaherty D (1984) The different types of limb apraxia errors made by patients with left vs. right hemisphere damage. Brain Cogn 3:370-384.

Haaland KY, Harrington DL, Knight RT (2000) Neural representations of skilled movement. Brain 123:2306-2313.

Haaland KY, Elsinger CL, Mayer AR, Durgerian S, Rao SM (2004) Motor sequence complexity and performing hand produce differential patterns of hemispheric lateralization. J Cogn Neurosci 16:621-636.

Kertesz A (1982) Western aphasia battery. New York: The Psychological Corporation.

Krakauer JW (2009) Motor learning and consolidation: The case of visuomotor rotation. Adv Exp Med Biol 629:405-421.

Krakauer JW, Ghilardi MF, Mentis M, Barnes A, Veytsman M, Eidelberg D, Ghez C (2004) Differential cortical and subcortical activations in the learning of rotations and gains for reaching: a PET study. J Neurophysiol 91:924-933.

Levin MF (1996) Interjoint coordination during pointing movements is disrupted in spastic hemiparesis. Brain 119:281-293.

Martin TA, Keating JG, Goodkin HP, Bastian AJ, Thach WT (1996) Throwing while looking through prisms. I. Focal olivocerebellar lesions impair adaptation. Brain 119:1183-1198.

McIntosh RD, Rossetti Y, Milner AD (2002) Prism adaptation improves chronic visual and haptic neglect: a single case study. Cortex 38:309-320.

Miall RC, Wolpert DM (1996) Forward models for physiological motor control. Neural Networks 9:1265-1279.

Mutha PK, Sainburg RL, Haaland KY (2010) Coordination deficits in ideomotor apraxia during visually targeted reaching reflect impaired visuomotor transformations. Neuropsychologia 48:3855-3867.

Newport R, Brown L, Husain M, Mort D, Jackson SR (2006) The role of the posterior parietal lobe in prism adaptation: Failure to adapt to optical prisms in a patient with bilateral damage to posterior parietal cortex. Cortex 42:720-729.

Oldfield RC (1971) The assessment and analysis of handedness: the Edinburgh Inventory. Neuropsychologia 9:97-113.

Oliveira FT, Diedrichsen J, Verstynen T, Duque J, Ivry RB (2010) Transcranial magnetic stimulation of posterior parietal cortex affects decisions of hand choice. Proc Natl Acad Sci U S A 107:17751-17756.

Pisella L, Rode G, Farnè A, Boisson D, Rossetti Y (2002) Dissociated long lasting improvements of straight-ahead pointing and line bisection tasks in two hemineglect patients. Neuropsychologia 40:327-334.

Pisella L, Michel C, Gréa H, Tilikete C, Vighetto A, Rossetti Y (2004) Preserved prism adaptation in bilateral optic ataxia: strategic versus adaptive reaction to prisms. Exp Brain Res 156:399-408.

Rorden C, Brett M (2000) Stereotaxic display of brain lesions. Behav Neurol 12:191-200

Rossetti Y, Rode G, Pisella L, Farné A, Li L, Boisson D, Perenin MT (1998) Prism adaptation to a rightward optical deviation rehabilitates left hemispatial neglect. Nature 395:166-169.

Rushworth MF, Nixon PD, Renowden S, Wade DT, Passingham RE (1997) The left parietal cortex and motor attention. Neuropsychologia 35:1261-1273.

Rushworth MF, Krams M, Passingham RE (2001) The attentional role of the left parietal cortex: the distinct lateralization and localization of motor attention in the human brain. J Cogn Neurosci 13:698-710.

Rushworth MF, Johansen-Berg H, Göbel SM, Devlin JT (2003) The left parietal and premotor cortices: motor attention and selection. Neuroimage 20 [Suppl 1]:S89-S100.

Sainburg RL (2005) Handedness: differential specializations for control of trajectory and position. Exerc Sport Sci Rev 33:206-213. 
Sainburg RL (2010) Lateralization of goal-directed movement. In: Vision and goal-directed movement: neurobehavioral perspectives (Elliott D, Khan M, eds), pp 219-238. Champaign, IL: Human Kinetics.

Sainburg RL, Schaefer SY (2004) Interlimb differences in control of movement extent. J Neurophysiol 92:1374-1383.

Schaefer SY, Haaland KY, Sainburg RL (2009) Dissociation of initial trajectory and final position errors during visuomotor adaptation following unilateral stroke. Brain Res 1298:78-91.

Shabbott BA, Sainburg RL (2010) Learning a visuomotor rotation: simultaneous visual and proprioceptive information is crucial for visuomotor remapping. Exp Brain Res 203:75-87.

Shadmehr R, Krakauer JW (2008) A computational neuroanatomy for motor control. Exp Brain Res 185:359-381.

Tanaka H, Sejnowski TJ, Krakauer JW (2009) Adaptation to visuomotor rotation through interaction between posterior parietal and motor cortical areas. J Neurophysiol 102:2921-2932.

Tseng YW, Diedrichsen J, Krakauer JW, Shadmehr R, Bastian AJ (2007) Sensory prediction errors drive cerebellum-dependent adaptation of reaching. J Neurophysiol 98:54-62.

Turton AJ, O’Leary K, Gabb J, Woodward R, Gilchrist ID (2010) A single blinded randomised controlled pilot trial of prism adaptation for improving self-care in stroke patients with neglect. Neuropsychol Rehabil 20:180-196.

Wolpert DM, Ghahramani Z (2000) Computational principles of movement neuroscience. Nat Neurosci 3:1212-1217.

Wolpert DM, Goodbody SJ, Husain M (1998) Maintaining internal representations: the role of the human superior parietal lobe. Nat Neurosci $1: 529-533$. 